\title{
Functional imaging using computer methods to compare the effect of salbutamol and ipratropium bromide in patient-specific airway models of COPD
}

\author{
This article was published in the following Dove Press journal: \\ International Journal of COPD \\ 25 November 2011 \\ Number of times this article has been viewed
}

\section{LA De Backer' \\ WG Vos ${ }^{2}$ \\ R Salgado ${ }^{3}$ \\ JW De Backer ${ }^{2}$ \\ A Devolder' \\ SL Verhulst' \\ R Claes' \\ PR Germonpré \\ WA De Backer'}

'Department of Respiratory Medicine, ${ }^{2}$ FluidDA, ${ }^{3}$ Department of Radiology, Antwerp University Hospital, Antwerp, Belgium
Correspondence: Lieve De Backer Department of Respiratory Medicine, Antwerp University Hospital, Wilrijkstraat 10, 2650 Edegem, Belgium

$\mathrm{Tel}+32382$ I 5480

$\mathrm{Fax}+3238214447$

Email Lieve.debacker@ua.ac.be
Background: Salbutamol and ipratropium bromide improve lung function in patients with chronic obstructive pulmonary disease (COPD). However, their bronchodilating effect has not yet been compared in the central and distal airways. Functional imaging using computational fluid dynamics offers the possibility of making such a comparison. The objective of this study was to assess the effects of salbutamol and ipratropium bromide on the geometry and computational fluid dynamics-based resistance of the central and distal airways.

Methods: Five patients with Global Initiative for Chronic Obstructive Lung Disease Stage III COPD were randomized to a single dose of salbutamol or ipratropium bromide in a crossover manner with a 1-week interval between treatments. Patients underwent lung function testing and a multislice computed tomography scan of the thorax that was used for functional imaging. Two hours after dosing, the patients again underwent lung function tests and repeat computed tomography.

Results: Lung function parameters, including forced expiratory volume in 1 second, vital capacity, overall airway resistance, and specific airway resistance, changed significantly after administration of each product. On functional imaging, the bronchodilating effect was greater in the distal airways, with a corresponding drop in airway resistance, compared with the central airways. Salbutamol and ipratropium bromide were equally effective at first glance when looking at lung function tests, but when viewed in more detail with functional imaging, hyporesponsiveness could be shown for salbutamol in one patient. Salbutamol was more effective in the other patients.

Conclusion: This pilot study gives an innovative insight into the modes of action of salbutamol and ipratropium bromide in patients with COPD, using the new techniques of functional imaging and computational fluid dynamics.

Keywords: chronic obstructive pulmonary disease, imaging, computed tomography, computational fluid dynamics, salbutamol, ipratropium

\section{Introduction}

Chronic obstructive pulmonary disease (COPD) is a heterogeneous disorder characterized by dysfunction of the small and large airways, as well as destruction of the lung parenchyma and its vasculature, in highly variable combinations. The hallmark of COPD is limitation of expiratory flow, which is slowly progressive and irreversible. ${ }^{1,2}$

Small airway function is still mainly evaluated using specific lung function tests, including flow-volume loops, nitrogen washout, and helium flow volume loops. ${ }^{3}$ Recently, there has been a trend towards the identification of new outcome parameters to evaluate 
lung function and treatment effects in COPD patients. In the literature, suggestions are made to investigate "volume parameters" like forced vital capacity after bronchodilation. ${ }^{4}$ However, these spirometric tests have their limitations, in that they are effort-dependent, and the deep inspiration and forced expiration required alone can lead to changes in airway tone. ${ }^{5}$ It is known that bronchodilator drugs can improve lung mechanics in COPD patients, despite little change in forced expiratory volume in 1 second $\left(\mathrm{FEV}_{1}\right)$, by decreasing the amount of hyperinflation. ${ }^{6}$ Also, advanced imaging techniques based on multislice high-resolution computed tomography (CT) have been used to characterize COPD patients. ${ }^{7}$ These techniques allow for evaluation of the density of the parenchyma ${ }^{8}$ or for evaluation of airway lumen and wall thickness. ${ }^{9,10}$ Effects of bronchodilation on the bronchus-vessel ratio and airway lumen caliber have been studied in COPD patients by De Luca et $\mathrm{al}^{11}$ and Hasegawa et al, ${ }^{12}$ respectively.

In recent years, a number of studies have used computational fluid dynamics to assess flow behavior in the respiratory system. The models used have been either idealized or based on realistic geometry, ${ }^{13-15}$ but very few studies have used image-based patient-specific models. ${ }^{16,17}$

Recent studies have indicated the possibility of using a combination of imaging tools and computer methods to provide functionality and to yield additional insight into clinically relevant issues, such as the bronchodilatory effects of inhaled medication. In the past, we have demonstrated a very good correlation between spirometric indices like $\mathrm{FEV}_{1}$ and Tiffeneau index $\left(\mathrm{FEV}_{1} /\right.$ vital capacity, an index to discriminate between obstructive and restrictive pathology) and computational fluid dynamics-based calculation of overall and distal airway resistance, ie, from the segmental airways onwards. Therefore, functional imaging can be used to assess functional changes in the distal airways after pharmacological intervention. With this technique, it is possible to make a comparison of the bronchodilation induced by salbutamol (Ventolin ${ }^{\mathrm{TM}}$, GlaxoSmithKline, London, UK) versus ipratropium bromide (Atrovent ${ }^{\circledR}$ HFA, Boehringer Ingelheim, Ingelheim, Germany).

Salbutamol, a short-acting beta ${ }_{2}$-agonist, induces bronchodilation for up to 6 hours. It is used to treat wheezing, dyspnea, and breathing difficulties caused by asthma and COPD, to prevent bronchospasm during exercise, and to improve lung function. ${ }^{18}$

Ipratropium bromide is a short-acting anticholinergic bronchodilator (muscarinic antagonist) that induces bronchodilation for up to 6 hours. Beneficial effects include improvement in lung function, wheezing, dyspnea, and health-related quality of life in patients with COPD. ${ }^{18}$
Comparisons between salbutamol and ipratropium bromide have been previously performed but limited to spirometric values and nonfunctional data, like dyspnea scores. In these studies, the degree of bronchodilation achieved with ipratropium bromide was equal to the degree of bronchodilation achieved after using a short-acting beta 2 -agonist. ${ }^{19}$ Both compounds improve exercise capacity and reduce dyspnea to a similar extent. ${ }^{20}$ It is as yet unclear if salbutamol has a different effect at the level of the peripheral airways in comparison with ipratropium bromide. ${ }^{18}$

The aim of this study was to compare the effects of salbutamol and ipratropium bromide at the level of all airways, especially in the distal and smaller airways. Recently developed functional imaging techniques offer the possibility to make these comparisons in detail and in a more sensitive way than is possible using the classical lung function tests. We compared these techniques in order to determine the most powerful method for assessment of bronchodilation in COPD.

\section{Materials and methods Patients}

We recruited five patients with severe but stable COPD (three men and two women with Global Initiative for Chronic Obstructive Lung Disease [GOLD] Stage III disease). The degree of airway obstruction was verified by a baseline pulmonary function test after at least 12 hours of bronchodilator withdrawal. Lung function testing was performed according to European Respiratory Society guidelines. ${ }^{21}$ The study protocol was approved by the local ethics committee and each patient gave informed consent to all procedures. Baseline patient characteristics are given in Table 1.

\section{Study design}

The study was an open, randomized, two-way crossover, pilot study involving a screening visit and 2 study days

Table I Baseline characteristics of the included patients with COPD

\begin{tabular}{lclll}
\hline & Mean \pm SD & Median & Minimum & Maximum \\
\hline Age (years) & $67.1 \pm 5.4$ & 65.6 & 61.6 & 76.2 \\
FEV,$(L)$ & $0.78 \pm 0.14$ & 0.81 & 0.60 & 0.93 \\
VC (L) & $2.38 \pm 0.92$ & 2.16 & 1.24 & 3.63 \\
FEV $/$ NC (\%) & $35.40 \pm 10.33$ & 34.00 & 22.00 & 48.00 \\
TLC (L) $_{\text {F }}$ & $6.63 \pm 1.79$ & 6.99 & 4.91 & 9.19 \\
FRC (L) & $5.11 \pm 1.49$ & 4.82 & 3.59 & 7.28 \\
Raw (kPa.s/L) & $1.12 \pm 0.17$ & 1.04 & 1.00 & 1.41 \\
\hline
\end{tabular}

Abbreviations: $\mathrm{FEV}_{1}$, forced expiratory volume in I second; VC, vital capacity; TLC, total lung capacity; SD, standard deviation; FRC, functional residual capacity. 
separated by at least 1 week to avoid any carryover effect. Prior to the first dosing, patients underwent lung function testing (spirometry, body plethysmography) and low-dose, high-resolution CT scanning of the thorax. After dosing, the patients once more underwent lung function tests and lowdose, high-resolution CT scanning. These measurements were done $90 \pm 30$ minutes after the dose. The assessments on the second study day were the same as those done on the first day, except that the CT pre-dose was not taken to limit radiation exposure.

\section{Study medication}

The study medications, salbutamol and ipratropium bromide, were supplied by GlaxoSmithKline and Boehringer Ingelheim, respectively. The original medication and packaging were used. At each visit, the patients received either four puffs of salbutamol $100 \mu \mathrm{g}$ or four puffs of ipratropium bromide $20 \mu \mathrm{g}$. The medication was administered by the investigator or by a member of the clinical unit designated by the investigator. Patients were trained to use the devices.

After gentle expiration, a dose of salbutamol $100 \mu \mathrm{g}$ or ipratropium bromide $20 \mu \mathrm{g}$ was inhaled in one breath to total lung capacity from a valved spacer device. The breath was then held for 5-10 seconds before the subject exhaled. Four separate doses (total dose $400 \mu \mathrm{g}$ of salbutamol or $80 \mu \mathrm{g}$ of ipratropium bromide) were delivered at 30 -second intervals.

Inhaled corticosteroids were permitted during the study, provided that the dose remained constant for at least 6 weeks prior to the first visit and remained constant throughout the study period. An appropriate washout period for bronchodilating products was determined to be at least 24 hours for long-acting bronchodilators and at least 6 hours for shortacting bronchodilators prior to both study days.

\section{$\mathrm{CT}$ and segmentation}

High-resolution CT scans of the thorax can detect airways in the respiratory system down to the small airways with a diameter of 1-2 mm when imaged at total lung capacity. ${ }^{22-25}$ From high-resolution CT images, patient-specific values can be derived for the lumen cross-sectional area, airway length, airway wall thickness, and the extent of emphysema. These values can be assessed either in a particular plane or by analyzing multiple slices over a defined longitudinal region. These high-resolution CT scans enable three-dimensional reconstruction of the airway geometry regions to be developed. This geometry reconstruction is performed by semiautomatic segmentation of the airways. This involves identification and grouping of voxels that belong to a specific anatomic structure, including the airway tree, the lungs or lobes or, in some cases, the fissures. A three-dimensional reconstruction of the morphology can then be performed. Outcome parameters include lung volumes, lobar volumes, and local airway volumes. By assessing multiple scans of the same patient, it is possible to analyze the changes in different parameters either over time (eg, before and after bronchodilation), or at several breathing levels, such as forced residual capacity or total lung capacity. In this study, at each CT data collection time point, two CT thorax scans were taken on a 64-slice GE LightSpeed VCT scanner. One scan was taken at total lung capacity, the other at functional residual capacity. Lung volumes were controlled using adapted spirometry during the high-resolution CT procedure. Scans were taken in a dose reduction protocol, ie, reduced tube voltage $(100 \mathrm{kV})$ and tube current as a function of patient weight $(1 \mathrm{mAs} / \mathrm{kg})$. In addition to this, there was an increase in noise factor to reduce the radiation dose further. Total radiation dose for the high-resolution CT scans taken in this study per patient was less than $12 \mathrm{mSv}$. Scanning time was less than 5 seconds per scan, voxel dimension was approximately $0.5 \mathrm{~mm}^{3}$, and the scanning region extended from the larynx down to the diaphragm.

From the high-resolution CT data, three-dimensional reconstruction of the airway geometry at total lung capacity was performed using semiautomatic segmentation of the airways up to the point where no distinction could be made between intraluminal and alveolar air (fifth to seventh bifurcation, airway diameter approximately $1 \mathrm{~mm}$ ). In addition, the lung lobes were segmented both at total lung capacity and functional residual capacity by identifying the fissure planes and subsequently using these surfaces as cutting objects. By segmenting the lung lobes at two lung levels it was possible to assess lobar expansion and hence the internal patient-specific mass flow distribution in the lower airways. The segmentation and three-dimensional model reconstruction was performed using a commercially developed and validated software package (Mimics $^{\circledR}$, Materialise, Leuven, Belgium). To determine the local change in airway volume, the models were subdivided into individual branches according to the nomenclature defined by Ikeda (as described in $\mathrm{Netter}^{26}$ ). For each individual airway branch, the volume $\left(\mathrm{iV}_{\mathrm{aw}}\right)$ was determined before and after the administration of the medication.

\section{Computational fluid dynamics}

Functionality can be added to the static segmented images by applying computational fluid dynamics methods to characterize airway resistance and aerosol deposition patterns. 
The postprocessing involves airway wall smoothing and reduction of segmentation artifacts. The airway model is then converted into a computational grid using a commercial software package $\left(\mathrm{T}-\mathrm{Grid}^{\circledR}\right.$, Fluent Inc, Lebanon, $\left.\mathrm{NH}\right)$ in order to solve the Navier-Stokes flow equations numerically. Flow is incompressible and isothermal, and most recent computational fluid dynamics models take into account the image-based internal flow distribution by including lobar expansion as a boundary condition. Computational fluid dynamics enables the determination of flow properties, including pressure, velocity, and density inside the entire flow domain. This allows the calculation of airway resistance by assessing the total pressure drop and airflow rate. Mesh size was around $4.5 \times 10^{6}$ cells. Steady flow simulations on these grids were performed using a commercial computational fluid dynamics solver (Fluent Inc). A mass flow of $30 \mathrm{~L} /$ minute was specified as the boundary condition at the trachea to reflect normal tidal breathing. At the outlets, the static pressure was defined using an iterative process to reflect the internal mass flow distribution matching the lobar expansion as derived from the CT data. The flow was considered laminar, incompressible, and adiabatic. Detailed descriptions of the segmentation and flow simulation procedures can be found in De Backer et al. ${ }^{24}$

From the computational fluid dynamics data, the $\mathrm{iR}_{\mathrm{aw}}$ was obtained. $\mathrm{iR}_{\mathrm{aw}}$ was defined as:

$$
\mathrm{iR}_{\mathrm{aw}}=\frac{\Delta p}{F}
$$

where $\Delta p$ is the total pressure drop over a certain region and $F$ is the mass flow rate of air through this region.

\section{Statistical analysis}

Comparisons of airway characteristics before and after medication, as well as changes in airway characteristics between both medications, were assessed using the Wilcoxon matched-pairs test.

Comparisons between pulmonary function tests and functional imaging were made using Spearman rank correlations. For all analyses, $P<0.05$ was defined as statistically significant and $P<0.1$ as a trend. Analyses were performed using Statistica ${ }^{\circledR}$ software (StatSoft, Sandton, South Africa).

\section{Results}

All five patients completed the study. The patients (three male and two female, mean age 67 years) had GOLD Stage III COPD, a mean 71 pack-year smoking history, and an average $\mathrm{FEV}_{1}$ of $35 \%$ predicted (Tables 1 and 2 ). A detailed overview of the results and the abbreviations used can be found in Table 3 and Figures 1-3.

For these five patients, there was a significant increase in vital capacity $(P=0.04)$ and decrease in airway resistance $\left(\mathrm{R}_{\mathrm{aw}}, P=0.04\right)$ and specific resistance $\left(\mathrm{sR}_{\mathrm{aw}}, P=0.04\right)$ 1.5 hours after inhalation of salbutamol. Functional imaging parameters showed an increase in total airway volume (iV $\mathrm{iV}_{\text {awtot }}, P=0.04$ ) after salbutamol, with a small rise in $\mathrm{iV}_{\text {awcentr }}$ $(P=0.08)$ and a large rise in $\mathrm{iV}_{\text {awdist }}(P=0.04)$. Also, after salbutamol, $\mathrm{iR}_{\text {awtot }}$ decreased $(P=0.04)$ due to a reduction in $\mathrm{iR}_{\text {awcentr }}(P=0.08)$ and $\mathrm{iR}_{\text {awdist }}(P=0.08)$.

After ipratropium bromide, there was a rise in $\mathrm{FEV}_{1}$ $(P=0.04)$ and a trend towards an increase in vital capacity $(P=0.08)$, and a decrease in $\mathrm{R}_{\text {aw }}(P=0.08)$ and $\mathrm{sR}_{\text {aw }}$ $(P=0.08)$. Functional imaging parameters show an increase in $\mathrm{iV}_{\text {awtot }}(P=0.04)$, with a small rise in $\mathrm{iV}_{\text {awcentr }}(P=0.04)$ and a large rise in $\mathrm{iV}_{\text {awdist }}(P=0.04)$. Also, after ipratropium bromide, $\mathrm{iR}_{\text {awtot }}$ decreased $(P=0.04)$ due to a decrease in $\mathrm{iR}_{\text {awcentr }}(P=0.08)$ and $\mathrm{iR}_{\text {awdist }}(P=0.08)$.

No significant differences were found between changes in lung function testing and functional imaging parameters

Table 2 Baseline lung function values in individual patients

\begin{tabular}{|c|c|c|c|c|c|c|c|c|c|}
\hline Patient & Age (years) & FEV, pre (\%) & FEV, post-BD (\%) & FEV, post-BD (L) & VC (L) & $\mathrm{FEV}_{\mathbf{l}} / \mathrm{VC}$ & TLC (L) & FRC (L) & $\mathbf{R}_{\mathrm{aw}}$ \\
\hline I & 66 & 31 & 35 & 0.73 & 1.95 & 34 & 7.14 & 5.87 & 1.04 \\
\hline 2 & 65 & 34 & 34 & 0.91 & 2.16 & 43 & 4.92 & 4 & 1.12 \\
\hline 3 & 76 & 31 & 37 & 0.71 & 1.24 & 48 & 4.91 & 3.59 & 1.409 \\
\hline 4 & 65 & 25 & 33 & 1.07 & 3.63 & 22 & 9.19 & 7.28 & 1.02 \\
\hline 5 & 61 & 33 & 36 & 0.98 & 2.92 & 30 & 6.99 & 4.82 & 1 \\
\hline Patient & RV (L) & IC (L) & & & & & & & \\
\hline I & 5.19 & 1.35 & & & & & & & \\
\hline 2 & 2.76 & 1.01 & & & & & & & \\
\hline 3 & 3.67 & 1.04 & & & & & & & \\
\hline 4 & 5.55 & 1.92 & & & & & & & \\
\hline 5 & 4.07 & 1.73 & & & & & & & \\
\hline
\end{tabular}

Abbreviations: BD, bronchodilator; RFC, residual functional capacity; RV, residual volume; IC, inspiratory capacity; FEV , forced expiratory volume in I second; TLC, total lung capacity; VC, vital capacity; $\mathrm{R}_{\mathrm{aw}}$, airway resistance 


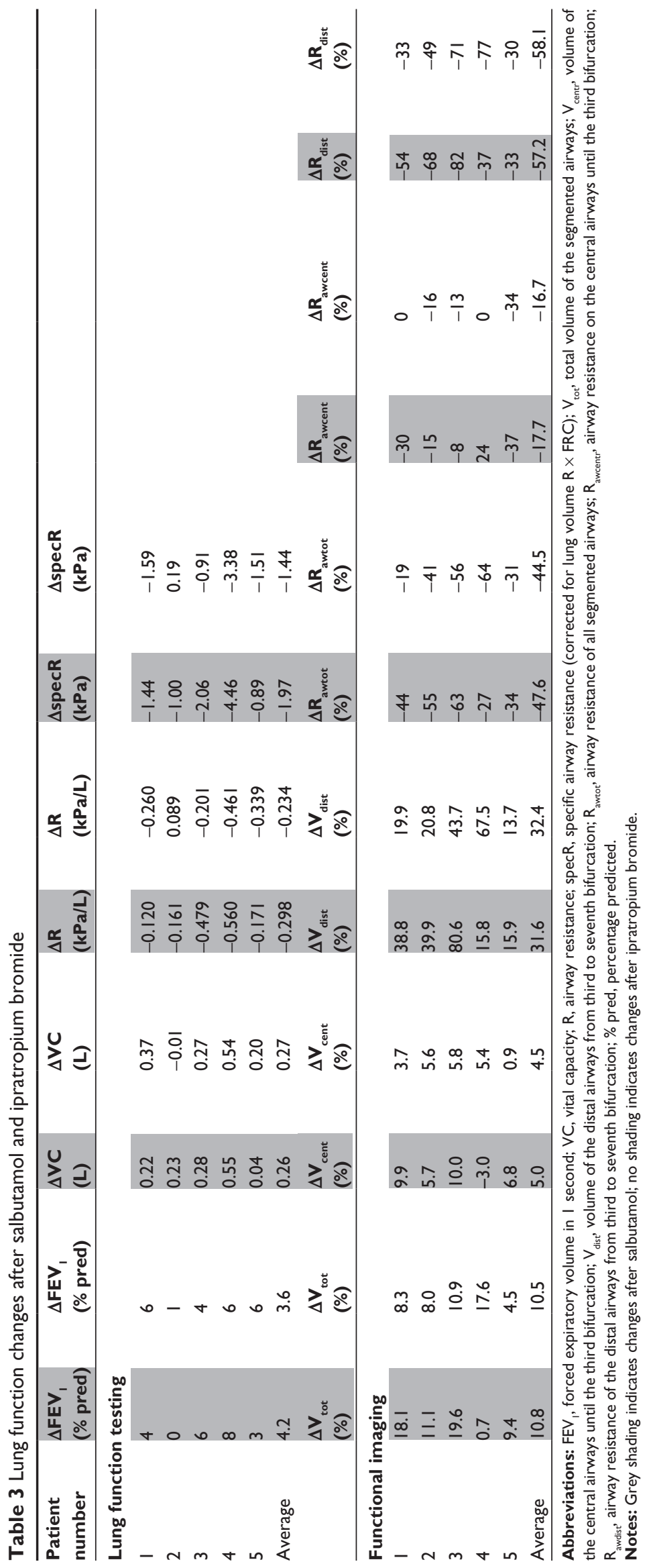



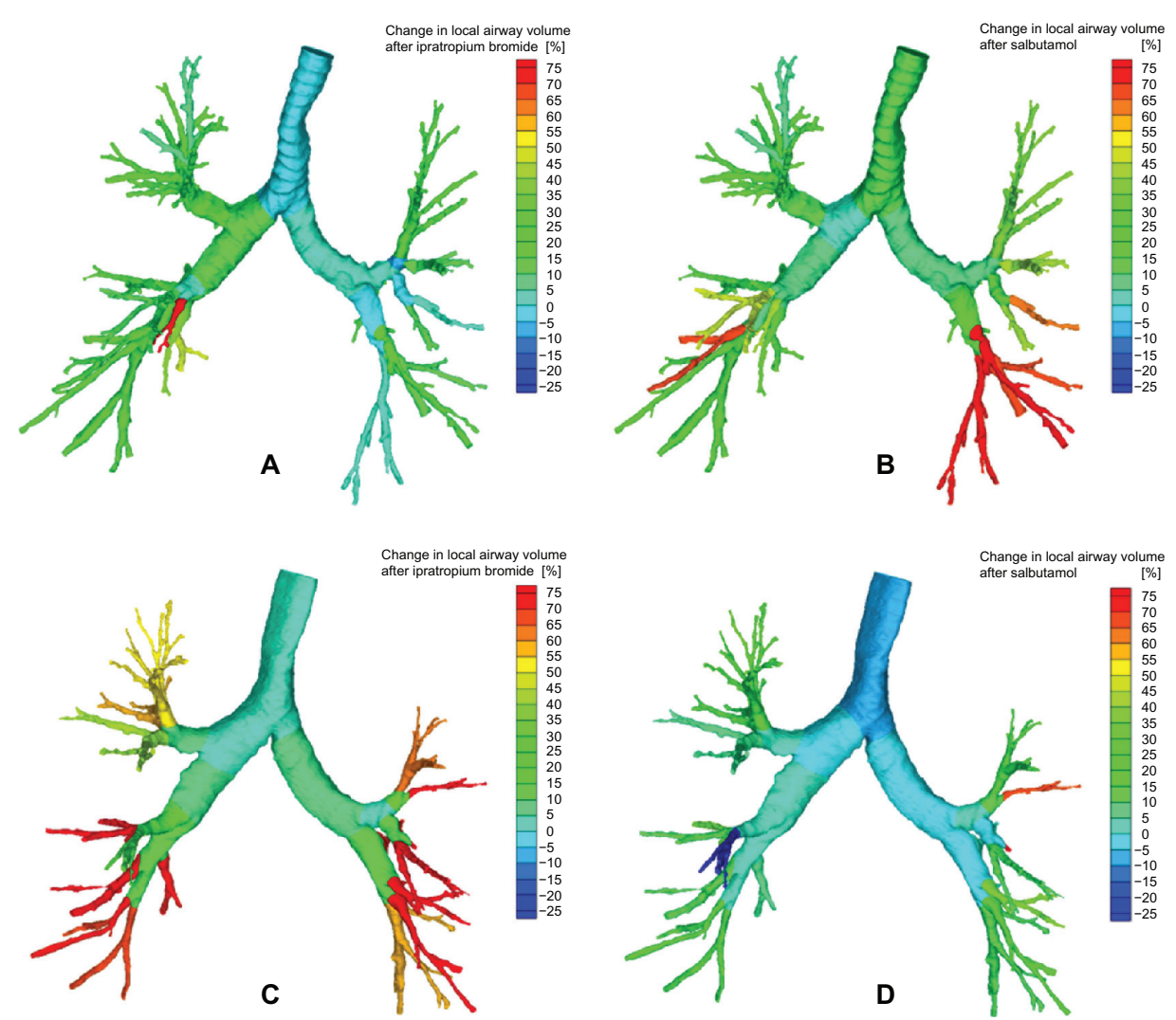

Figure I Changes in airway volume based an segmented airways from the CT scan (iVtot) in a patient (upper figures) mainly responsive to salbutamol (B) and less to ipratropium bromide (A), and in a patient (lower figures) more responsive to ipratropium bromide (C) and less responsive to salbutamol (D).
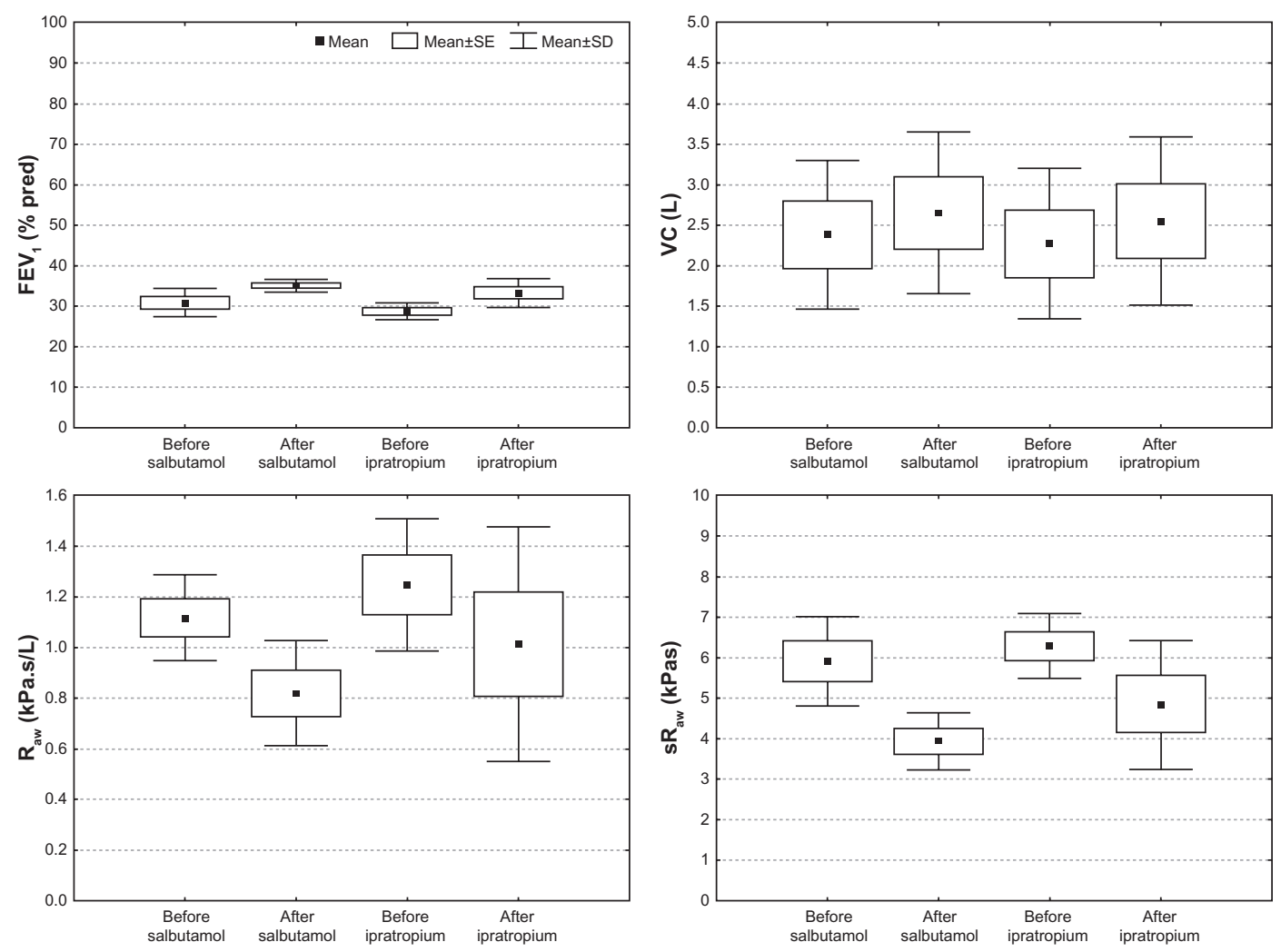

Figure 2 Changes in FEV, VC, Raw and sR $\mathrm{aw}_{\text {aw }}$ after salbutamol and ipratropium in five COPD patients (GOLD III). All changes are significant $(P<0.05)$ except the changes in $\mathrm{FEV}$, after salbutamol and $\mathrm{VC}$ after ipratropium.

Abbreviations: FEV , forced expiratory volume in I second; VC, vital capacity; SE, standard error; SD, standard deviation. 

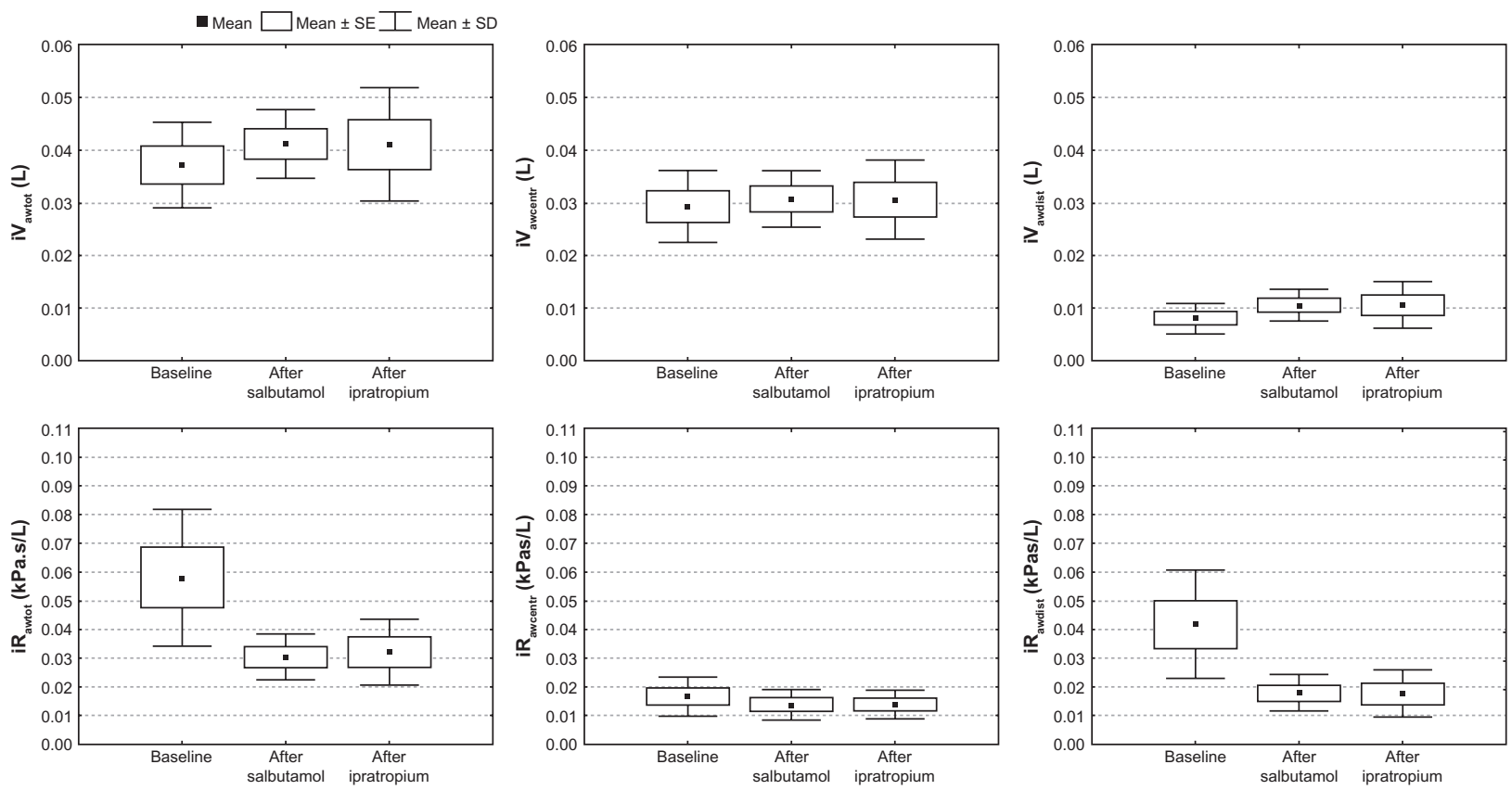

Figure 3 Changes after salbutamol and ipratropium bromide in total $\left(i \mathrm{~V}_{\text {too }}\right)$, central $\left(\mathrm{i} \mathrm{V}_{\text {centr }}\right)$ and distal airway volume $\left(\mathrm{i} \mathrm{V}_{\text {dis }}\right)$ based on images of segmented airways and corresponding resistance ( $\mathrm{R}_{\text {awtor }}, \mathrm{i}_{\text {awcentr }}$ and $\mathrm{R}_{\text {awdiss }}$ ) using computational fluid dynamics, in five COPD patients (GOLD ST III). All changes are significant $(P<0.05)$ except for $\mathrm{i}_{\text {awcentr }}$ after salbutamol and $\mathrm{iR}_{\text {awcentr }}$ after both salbutamol and ipratropium bromide.

Abbreviations: SD, standard deviation; SE, standard error.

after salbutamol or after ipratropium bromide. Looking at the functional imaging parameters, we noticed a trend towards a higher response to salbutamol. However, for one patient, we observed an inverse trend, with hyporeactivity to salbutamol. Repeating the statistical analysis without this hyporesponder to salbutamol, we obtain a trend in favor of salbutamol (compared with ipratropium bromide) for the change in $\mathrm{iV}_{\text {autot }}$ $(P=0.07)$ and $\mathrm{iR}_{\text {awtot }}(P=0.07)$. Lung function testing did not detect any differences between salbutamol and ipratropium bromide. When we compared functional radiologic measurements with classical lung function measurements (Tables 4 and 5), we only found a significant Spearman rank correlation between resistance and $\mathrm{R}_{\mathrm{tot}}$. Functional imaging detects small differences and is more sensitive, which explains why these correlations are not strong.

\section{Discussion}

Using functional imaging, we demonstrated that central but mainly distal airway dimensions (third to seventh bifurcation) increased significantly after an inhaled beta ${ }_{2}$-agonist (salbutamol) and anticholinergic agent (ipratropium bromide) in patients with severe COPD. Corresponding changes in lung function (spirometry, airway resistance) could be observed, but the changes seen on lung function testing were overall less pronounced than the changes in airway caliber seen by the imaging method. The changes observed with imaging were even greater when airway resistance calculated using computational fluid dynamics was taken into consideration. The latter method seems to provide added, more pronounced, and more sensitive information in the calculation of airway dimensions. Also, discrepancies between $\mathrm{FEV}_{1}$ and forced vital capacity responses were observed, with more pronounced overall volume responses. Overall, the response to salbutamol was more pronounced than that to ipratropium bromide. However, ipratropium bromide was more active in one patient, especially when changes in airways caliber were taken into account.

Assessment of reversibility is recognized as an essential part of the management of airway obstruction. ${ }^{27}$ COPD patients are considered to have airflow limitation that is not fully reversible. While some patients can still show high reversibility, most COPD patients have limited changes in $\mathrm{FEV}_{1}$ after bronchodilators. ${ }^{28}$ Some can show larger changes in forced vital capacity and develop the so-called

Table 4 Spearman rank comparison between functional imaging resistance and pulmonary function test (PFT) resistance

\begin{tabular}{llll}
\hline & $\mathbf{R}_{\text {tot }}$ & $\mathbf{R}_{\text {awcentr }}$ & $\mathbf{R}_{\text {awdist }}$ \\
\hline$R($ PFT) & 0.52 & 0.17 & -0.21 \\
$P$ & 0.02 & 0.5 & 0.4 \\
Rspec (PFT) & 0.27 & -0.38 & 0.37 \\
$P$ & 0.3 & 0.09 & 0.1 \\
\hline
\end{tabular}

Abbreviations: $R_{\text {tot }}$, total airway resistance; $R_{\text {awcentr }}$, airway resistance in the central airways until third bifurcation; $R_{\text {awdist }}$ airway resistance of distal airways from third to seventh bifurcation. 
Table 5 Spearman rank comparison between change in pulmonary function test volumes and change in functional imaging volumes

\begin{tabular}{llll}
\hline & $\Delta \mathbf{V}_{\text {tot }}$ & $\Delta \mathbf{V}_{\text {centr }}$ & $\Delta \mathbf{V}_{\text {dist }}$ \\
\hline$\Delta \mathrm{RV}(\mathrm{L})$ & -0.32 & -0.08 & -0.29 \\
$P$ & 0.04 & 0.8 & 0.4 \\
$\Delta \mathrm{RV}(\%)$ & -0.46 & -0.26 & -0.39 \\
$P$ & 0.2 & 0.5 & 0.3 \\
$\Delta \mathrm{FRC}$ & -0.46 & -0.26 & -0.39 \\
$P$ & 0.2 & 0.5 & 0.3 \\
\hline
\end{tabular}

Abbreviations: $R V$, residual volume; $F R C$, functional residual capacity; $V_{\text {tor }}$ total volume of the segmented airways; $\mathrm{V}_{\text {centr }}$, volume of the central airways until the third bifurcation.

volume response ${ }^{29}$ which is seen more in severe patients with end-expiratory flow limitation..$^{4-30} \mathrm{We}$ focused on the more common, severe COPD patients with little reversibility in $\mathrm{FEV}_{1}$ after bronchodilators. The functional imaging used in this study provided us with the opportunity to see whether changes in airway caliber and calculated airway resistance using computer methods were comparable with the limited changes seen in $\mathrm{FEV}_{1}$ in these patients. Moreover, the imaging method used provided information on changes in both central (until the third bifurcation) and distal (third to seventh bifurcation) airways. High-resolution CT has been used previously to determine bronchodilator responses either looking at changes in parenchymal density ${ }^{31}$ or at bronchus-vessel caliber relationship. ${ }^{11}$ Both methods are more indirect than the segmentation method used in this study complemented with computational fluid dynamics-based airway resistance measurements. We did not calculate emphysema scores for these patients, but we know from as yet unpublished data that functional imaging can be of value in doing this, because we can measure blood vessel density in every lobe and compare this with the percentage of airflow going to this lobe. This way, we have an idea about the ventilation/perfusion match. We certainly have to include this technique in a larger study in the future.

The variable response to different bronchodilators in COPD patients has been observed before, and previous attempts to identify the right patients for certain products were not satisfying. Oga et $\mathrm{al}^{20}$ found that the effects of different bronchodilators on exercise capacity varied among individuals with stable COPD, while the degree of bronchodilation measured by $\mathrm{FEV}_{1}$ did not correlate with the increase in endurance time of the exercise test. This suggests that $\mathrm{FEV}_{1}$ is not an optimal descriptor of the bronchodilator effect. We did not measure diffusing capacity of the lung for carbon monoxide or maximum inspiratory and expiratory pressure in this study, which could be interesting to compare in larger studies in the future, as would be the BODE index (we did not measure the 6-minute walking distance), because it is a global measure of COPD severity, which takes into account some important health-related quality of life measures.

We found that the response to salbutamol was larger in four out of five of our patients compared with the response to ipratropium bromide. However, a hyporesponse to salbutamol was observed for one patient. Classical lung function testing was not able to make this distinction. $\mathrm{FEV}_{1}$ clearly underestimates the acute bronchodilator effects in COPD patients, ${ }^{32}$ probably because the main effect is situated in the distal airways, as we see with imaging.

In this study, we used functional imaging as a method for evaluating bronchodilator treatment in COPD. Because COPD is such a heterogeneous disease, the same treatment does not have the same effect in different patients. This method helps us to understand why this is the case. It is the first method to evaluate regional resistances and volumes, and gives us new insights into the mechanism of bronchodilating treatment. It could help us to select the right treatment for the right patient, which should be the goal of every physician.

Suboptimal dosing may contribute to the wide variation of responders with different degrees of reversibility, because dose-response relationships have been demonstrated. ${ }^{33}$ Using higher doses, there is no substantial difference between using a short-acting beta-agonist or a short-acting muscarinic agonist. It has also been demonstrated that there is an effective improvement in $\mathrm{FEV}_{1}$ on adding a high dose of salbutamol $(600 \mu \mathrm{g})$ or a high dose of ipratropium bromide $(120 \mu \mathrm{g})$ to regular tiotropium. ${ }^{34} \mathrm{~A}$ limitation of this study is the fact that we only studied patients with more severe GOLD Stage III COPD who are known to have more volume response. Additional insight could be gained by performing the same tests on patients with GOLD Stage I, II, and IV COPD.

Different descriptors of the disease, like genetics and emphysema scores, might also be of help in predicting response. Some studies have shown that genotype may determine the bronchodilating response. ${ }^{35,36} \mathrm{~A}$ larger study including all degrees of severity and performed over a longer time period could also give important information on the relationship between clinical outcomes (quality of life, dyspnea scores) and imaging data. We can conclude that salbutamol and ipratropium bromide have a bronchodilating effect in COPD patients, as indicated by a significant improvement in $\mathrm{FEV}_{1}$, vital capacity, $\mathrm{R}_{\mathrm{aw}}$, and $s \mathrm{R}_{\mathrm{aw}}$ in 
classical lung function testing. Using functional imaging, regional differences in airway volumes and resistances can be detected after bronchodilation. Distal bronchodilation is more pronounced than central bronchodilation. $\mathrm{iR}_{\text {awtot }}$ decreases, with a redistribution of airway resistance from the distal to the central airways. From classical lung function tests, there seems to be no difference in efficiency between salbutamol and ipratropium bromide. Nevertheless, using functional imaging parameters, we can distinguish two different types of responders, ie, the ones who respond more to salbutamol and a possible subgroup of hyporesponders to salbutamol. To confirm these findings, we need to study a larger group of patients, looking at different characteristics to predict and explain these different responses.

\section{Disclosure}

The authors report no conflicts of interest in this work.

\section{References}

1. Bosken CH, Wiggs BR, Pare PD, Hogg JC. Small airway dimensions in smokers with obstruction to airflow. Am Rev Respir Dis. 1990;142(3):563-570.

2. Kuwano K, Bosken CH, Pare PD, Bai TR, Wiggs BR, Hogg JC. Small airways dimensions in asthma and in chronic obstructive pulmonary disease. Am Rev Respir Dis. 1993;148(5):1220-1225.

3. Pare PD, Brooks LA, Coppin CA, et al. Density-dependence of maximal expiratory flow and its correlation with small airway disease in smokers. Am Rev Respir Dis. 1985;131(4):521-526.

4. Walker PP, Calverley PM. The volumetric response to bronchodilators in stable chronic obstructive pulmonary disease. COPD. 2008;5(3):147-152.

5. Burns GP, Gibson GJ. A novel hypothesis to explain the bronchconstrictor effect of deep inspiration in asthma. Thorax. 2002;57(2): 116-119.

6. Newton MF, O’Donnell DE, Forkert L. Response of lung volumes to inhaled salbutamol in a large population of patients with severe hyperinflation. Chest. 2002;121(4):1042-1050.

7. Grenier PA, Beigelman-Aubry C, Fetita C, Preteux F, Brauner MW, Lenoir S. New frontiers in CT imaging of airway disease. Eur Radiol. 2002;12(5):1022-1044.

8. Gevenois PA, Yernault JC. Can computed tomography quantify pulmonary emphysema? Eur Respir J. 1995;8(5):843-848.

9. Achenbach T, Weinheimer O, Biedermann A, et al. MDCT assessment of airway wall thickness in COPD patients using a new method: Correlations with pulmonary function tests. Eur Radiol. 2008;18(12):2731-2738.

10. Brillet PY, Fetita CI, Saragaglia A, et al. Investigation of airways using MDCT for visual and quantitative assessment in COPD patients. Int $J$ Chron Obstruct Pulmon Dis. 2008;3(1):97-107.

11. De Luca N, Capuzi P, D’Angeli AL, et al. High resolution computed tomography (HRCT) assessment of beta 2-agonist induced bronchodilation in chronic obstructive pulmonary disease patients. Eur Rev Med Pharmacol Sci. 1999;3(2):83-87.

12. Hasegawa M, Makita H, Nasuhara Y, et al. Relationship between improved airflow limitation and changes in airway calibre induced by inhaled anticholinergic agents in COPD. Thorax. 2009;64(4): $332-338$.
13. Longest PW, Vinchurkar S. Effects of mesh style and grid convergence on particle deposition in bifurcating airway models with comparisons to experimental data. Med Eng Phys. 2007;29(3):350-366.

14. Yang XL, Liu Y, So RM, Yang JM. The effect of inlet velocity profile on the bifurcation COPD airway flow. Comput Biol Med. 2006;36(2):181-194.

15. Ma B, Lutchen KR. CFD simulation of aerosol deposition in an anatomically based human large-medium airway model. Ann Biomed Eng. 2009;37(2):271-285.

16. de Rochefort L, Vial L, Fodil R, et al. In vitro validation of computational fluid dynamic simulation in human proximal airways with hyperpolarized ${ }^{3} \mathrm{He}$ magnetic resonance phase-contrast velocimetry. J Appl Physiol. 2007;102(5):2012-2023.

17. Lin CL, Tawhai M, McLennan G, Hoffman E. Computational fluid dynamics. IEEE Eng Med Biol Mag. 2009;28(3):25-33.

18. Gross NJ. Anticholinergic agents in asthma and COPD. Eur J Pharmacol. 2006;533(1-3):36-39.

19. McCrory DC, Brown CD. Anti-cholinergic bronchodilators versus beta2-sympathomimetic agents for acute exacerbations of chronic obstructive pulmonary disease. Cochrane Database Syst Rev. 2002;4:CD003900.

20. Oga T, Nishimura K, Tsukino M, Sato S, Hajiro T, Mishima M. A comparison of the effects of salbutamol and ipratropium bromide on exercise endurance in patients with COPD. Chest. 2003;123(6):1810-1816.

21. Brusasco V, Crapo R, Viegi G. Coming together: The ATS/ERS consensus on clinical pulmonary function testing. Eur Respir J. 2005;26(1):1-2.

22. De Backer JW, Vos WG, Vinchurkar SC, et al. Validation of computational fluid dynamics in CT-based airway models with SPECT/CT. Radiology. 2010;257(3):854-862.

23. De Backer JW, Vos WG, Burnell P, et al. Study of the variability in upper and lower airway morphology in Sprague-Dawley rats using modern micro-CT scan-based segmentation techniques. Anat Rec (Hoboken). 2009;292(5):720-727.

24. De Backer JW, Vos WG, Gorle CD, et al. Flow analyses in the lower airways: Patient-specific model and boundary conditions. Med Eng Phys. 2008;30(7):872-879.

25. De Backer JW, Vos WG, Devolder A, et al. Computational fluid dynamics can detect changes in airway resistance in asthmatics after acute bronchodilation. J Biomech. 2008;41(1): 106-113.

26. Netter FH. Respiratory System. Summit, NJ: CIBA Collection; 1979.

27. Calverley PM, Burge PS, Spencer S, Anderson JA, Jones PW. Bronchodilator reversibility testing in chronic obstructive pulmonary disease. Thorax. 2003;58(8):659-664.

28. Tashkin DP, Celli B, Decramer M, et al. Bronchodilator responsiveness in patients with COPD. Eur Respir J. 2008;31(4):742-750.

29. Ben SH, Prefaut C, Tabka Z, Zbidi A, Hayot M. The forgotten message from GOLD: FVC is a primary clinical outcome measure of bronchodilator reversibility in COPD. Pulm Pharmacol Ther. 2008;21(5):767-773.

30. Schermer T, Heijdra Y, Zadel S, et al. Flow and volume responses after routine salbutamol reversibility testing in mild to very severe COPD. Respir Med. 2007;101(6):1355-1362.

31. Kurashima K, Takayanagi N, Sato N, et al. High resolution CT and bronchial reversibility test for diagnosing COPD. Respirology. 2005;10(3):316-322.

32. Borrill ZL, Houghton CM, Woodcock AA, Vestbo J, Singh D. Measuring bronchodilation in COPD clinical trials. Br J Clin Pharmacol. 2005;59(4):379-384.

33. Teale C, Morrison JF, Page RL, Pearson SB. Dose response to inhaled salbutamol in chronic obstructive airways disease. Postgrad Med J. 1991;67(790):754-756.

34. Cazzola M, Santus P, D’Adda A, Pizzolato S, DiMarco F, Centanni S. Acute effects of higher than standard doses of salbutamol and ipratropium on tiotropium-induced bronchodilation in patients with stable COPD. Pulm Pharmacol Ther. 2009;22(3):177-182. 
35. Kim WJ, Hersh CP, DeMeo DL, Reilly JJ, Silverman EK. Genetic association analysis of COPD candidate genes with bronchodilator responsiveness. Respir Med. 2009;103(4):552-557.
36. Umeda N, Yoshikawa T, Kanazawa H, Hirata K, Fujimoto S. Association of beta2-adrenoreceptor genotypes with bronchodilatory effect of tiotropium in COPD. Respirology. 2008;13(3):346-352.

\section{Publish your work in this journal}

The International Journal of COPD is an international, peer-reviewed journal of therapeutics and pharmacology focusing on concise rapid reporting of clinical studies and reviews in COPD. Special focus is given to the pathophysiological processes underlying the disease, intervention programs, patient focused education, and self management protocols.
This journal is indexed on PubMed Central, MedLine and CAS. The manuscript management system is completely online and includes a very quick and fair peer-review system, which is all easy to use. Visit http://www.dovepress.com/testimonials.php to read real quotes from published authors. 